\title{
Required pecking alters judgments of the passage of time by pigeons
}

\author{
THOMAS R. ZENTALL, ANDREA M. FRIEDRICH, and TRICIA S. CLEMENT \\ University of Kentucky, Lexington, Kentucky
}

\begin{abstract}
There is evidence that how humans perceive time is affected by the activity in which they are engaged when they are judging time. In humans, typically, the more demanding the task, the faster time seems to pass. We asked whether a similar effect could be found in pigeons. Pigeons were trained to discriminate between a short- (2-sec) and a long- (10-sec) duration stimulus. Depending on the color of the stimulus (white or blue), the pigeons were required to peck (at least once per second or the trial was aborted) or to refrain from pecking (pecks aborted the trial). Once these tasks had been acquired to a high degree, probe trials involving white and blue stimuli were presented at durations between 2 and 10 sec. On trials in which the pigeons were required to peck, the point of subjective equality (i.e., the point at which pigeons are equally likely to choose the stimulus associated with long stimuli as the stimulus associated with short stimuli) was almost $1 \mathrm{sec}$ longer than on trials in which the pigeons were required to refrain from pecking. In other words, on trials that required pecking, more time passed before the pigeons indicated that the probe duration was at the subjective midpoint between 2 and $10 \mathrm{sec}$ than on trials that did not require pecking. This result suggests that like humans, the pigeons underestimated the passage of time when they were active or when attention to time-related cues had to be shared with attention to satisfying the response rate requirement.
\end{abstract}

It is said that time flies when one is having fun (or more likely when one is attending to things other than time). Conversely, every undergraduate knows how slowly time passes during an uninteresting lecture. Chastain and Ferraro (1997) have suggested that in humans, the subjectivity of estimates of time durations may reflect the nature of the cognitive operations occurring during the estimates. The greater the cognitive load, the greater the underestimation of the passage of time, whether the intervals being judged are very short (such as fractions of a second; see Chastain \& Ferraro, 1997) or much longer (such as minutes; see Hoekstra, 2005).

This effect occurs both retroactively, when subjects are asked to judge the passage of time after the fact, and proactively, when they are told in advance that they will be asked to judge the duration of an interval (Block \& Zakay, 1997). According to Chaston and Kingstone (2004), the subjective compression of time when one is engaged in a demanding task results from the relative inattention given to cues provided by the internal clock (see also Church, 1978); specifically, they suggest that the passage of time is judged by the accumulation of pulses coming from a pulse generator (pacemaker). Chaston and Kingstone proposed that if attention is not directed at the timing of an interval due to the cognitive demands of a task, it is because either

This research was supported by National Institute of Mental Health Grant MH-63726. Correspondence concerning this article should be sent to T. R. Zentall, Department of Psychology, University of Kentucky, Lexington, KY 40506-0044 (e-mail: zentall@uky.edu). the pulse output is slowed or some of the pulses may have been lost (see also Zakay, 1993). In either case, the accumulation of pulses will be less than the time that has actually elapsed, resulting in an underestimation of the passage of time.

The notion of an internal clock that is used to time intervals has also been extended to account for interval timing in animals (see, e.g., Gibbon \& Church, 1984). If attention to the internal clock can also affect time judgments by animals, then one might expect the demands of a task to similarly affect the animals' estimation of an interval's duration.

Pigeons have often been used in experiments in which reinforcement either occurs at an expected time (a fixed interval schedule) or does not occur (extinction); a pigeon's peak rate of responding at the expected time is taken as a measure of its ability to judge the duration of the interval (Roberts, Cheng, \& Cohen, 1989).

Pigeons have also been used in conditional discrimination experiments in which they must determine which of two (sample) stimulus durations has occurred, a short interval or a long interval (Stubbs, 1976). This procedure has been used to help describe the nature of pigeons' underlying timing scale. To determine the scale of time, pigeons are trained with a short (e.g., $2 \mathrm{sec}$ ) sample stimulus and a long (e.g., $8 \mathrm{sec}$ ) sample stimulus. Responses to one test (comparison) stimulus are reinforced following the short sample and responses to the other comparison stimulus are reinforced following the long sample. On test trials, sample stimuli of various intermediate durations are presented and the proportion of "long" choices is plotted at 
each test duration. The resulting psychophysical function indicates the degree to which each intermediate sample stimulus duration is judged as short or long.

When describing the underlying time scale, the point of subjective equality (the sample duration at which the pigeons are as likely to choose the comparison stimulus that in training was associated with short sample stimuli as the comparison stimulus associated with long sample stimuli) is of particular interest. The typical finding is that the point of subjective equality falls very close to the geometric rather than the arithmetic mean of the training durations (Gibbon, 1986; Platt \& Davis, 1983; Stubbs, 1976). In the example given, with samples of 2 and $8 \mathrm{sec}$, the geometric mean would be 4 sec.

One way to assess the effect of certain variables on the subjective judgment of time passage would be to look for a shift in the point of subjective equality. If subjective time were passing slowly for pigeons relative to actual time, then on a plot of choices of the comparison associated with long samples, the point of subjective equality would shift to a higher value (i.e., a longer sample duration), because the pigeons would be choosing "short" more than "long" when the test-trial sample duration was equal to the geometric mean. However, when we judge the passage of time as being shorter than it actually is, we say that time passed more quickly, or that more time has elapsed than we thought. Thus, when subjective time passes slowly, it means that actual time passes quickly. Whether time seems to pass slowly or quickly depends on the perspective from which the judgment is being made. For example, a student taking an examination might look at his watch and say, "Have $\mathbf{4 5}$ minutes passed already? I thought it was more like 30 minutes." Subjective time has slowed relative to actual time, but we would say that time has passed quickly.

The purpose of the present experiment was to determine if adding a response requirement that necessitated additional attention or created a heavier cognitive load while pigeons were discriminating between short and long intervals would result in a shift in their point of subjective equality. The rationale was that imposing a strict response requirement (as compared with no response requirement) on pigeons would be analogous to imposing a higher information processing or attentional load on humans. If attention to the response requirement (or the task load) had an effect on time judgments that was similar to that reported for humans, the pigeons would show a shift in the point of subjective equality to longer sample durations as compared with the point of subjective equality in a control condition for which the response requirement required less attention (or was less demanding). Thus, it is hypothesized that when the pigeons have to fulfill a response requirement while judging time, actual time should pass quickly relative to subjective time.

The pigeons in the present experiment were trained on two duration-sample matching tasks. Each task involved samples of 2 and $10 \mathrm{sec}$. One task was signaled by a white stimulus on the center response button, which for one group was followed by red and green comparison stimuli; the other task was signaled by a blue stimulus on the center response button, which was followed by a circle and a plus comparison stimulus. The important difference between the two tasks was the response requirement to the samples. In the task thought to be more demanding, the pigeons were required to peck at a minimum rate of 1 peck per second to both the short and the long sample. In the task thought to be less demanding, they were required to refrain from pecking for the duration of sample.

\section{METHOD}

\section{Subjects}

The subjects were 12 White Carneau pigeons (Columba livia) of unknown sex obtained from the Palmetto Pigeon Plant (Sumter, SC). The pigeons had previously participated in matching-to-sample experiments but had no experience in experiments that involved the timing of events. The pigeons were individually housed in a colony room maintained under a 12:12-h light:dark cycle. They were maintained at $85 \%$ of their free-feeding weights for the duration of the experiment, but had free access to water and grit in their home cages. The pigeons were cared for in accordance with University of Kentucky animal care guidelines.

\section{Apparatus}

The experiment was conducted in a standard test chamber (BRS/ LVE, Inc., Laurel, MD) that measured $33 \mathrm{~cm}$ high and $35 \mathrm{~cm}$ from response panel to back wall; the response panel measured $31 \mathrm{~cm}$ across. The response panel contained three horizontally aligned response buttons ( $2.5 \mathrm{~cm}$ in diameter, $8 \mathrm{~cm}$ apart center to center), which could be illuminated by projectors (Industrial Electronics Engineering, Model 10, Van Nuys, CA) mounted behind them. A houselight located above the center response button provided general illumination during intertrial intervals. All of the events in the chamber were controlled by a microcomputer located in an adjacent room. Reinforcement consisted of 1.5 sec of access to mixed grain (Purina Pro Grain) from an illuminated feeder located below the center response button.

\section{Procedure}

Pretraining. Initially, all the pigeons were trained to peck at the red, green, circle, and plus stimuli projected on the side response buttons. Then 6 pigeons were trained to peck at a white stimulus presented on the center response button, and the peck requirement was gradually increased to a fixed ratio (FR) of 10 pecks. The remaining 6 pigeons received FR10 training with a blue stimulus. Pretraining with the center response button continued for 144 trials or until the pigeons reliably completed the FR10 schedule.

Training. On each trial, white or blue samples could be presented for either 2 or $10 \mathrm{sec}$. The 6 pigeons pretrained to peck the white stimulus were required to make a minimum of 2 pecks per each 2 -sec white sample ( 24 trials per session) and a minimum of 10 pecks per each 10 -sec white sample ( 24 trials per session). Failure to meet the requirement led to the termination of the trial, followed by a $10-\mathrm{sec}$ intertrial interval and the restart of the trial, with a maximum of five repeats per trial. Completion of the sample requirement was followed by the presentation of red and green comparisons. Pigeons received reinforcement when they chose the red comparison on 2-sec sample trials and the green comparison on 10-sec sample trials.

On blue sample trials, the same pigeons were required to refrain from pecking (known as differential reinforcement of other behavior, or DRO) for $2 \mathrm{sec}$ on 2-sec sample trials (24 trials per session) and to refrain from pecking for $10 \mathrm{sec}$ on 10-sec sample trials (24 trials per session). The absence of pecking on these trials led to the presentation of a circle on one side response button and a plus on the other side response button as comparisons. Pigeons received 
reinforcements when they chose the circle on 2-sec sample trials and the plus on 10-sec sample trials. Failure to refrain from pecking led to the immediate termination of the trial followed by the intertrial interval and a maximum of five repeats per trial.

For the remaining 6 pigeons, which were pretrained to peck at a blue stimulus, the response requirements (pecking or refraining from pecking) for the white and blue sample trials were reversed, but the comparison stimuli following white and blue samples continued to be red and green and circle and plus, respectively. In all other respects, all the pigeons were treated the same.

Training sessions consisted of 96 trials: 24 each of the 2- and 10 -sec white trials and 24 each of the 2- and 10-sec blue trials.

Training trials were presented in random order, and all trials were followed by a $10-\mathrm{sec}$ illuminated intertrial interval. Nonreinforced choice trials also led to a repetition of the trial, and each trial could be repeated up to five times. This correction procedure was removed when $80 \%$ overall accuracy was achieved. A schematic representation of the training events for pigeons trained to peck the white stimulus appears in Figure 1. Training continued for each pigeon until it exhibited a stable level of matching accuracy on each of the four training trial types and attained an overall level of matching accuracy of at least $90 \%$ in one training session. One pigeon was dropped from the study for failure to reach this level of matching accuracy.

Testing. Testing sessions consisted of an additional 48 training trials and 56 test trials. During continued training trials, the training contingencies were in effect. On test trials, a blue or white sample was presented for $3,4,5,6,7,8$, or $9 \sec$ (four trials of each color at each stimulus duration). There was no pecking requirement on these test trials, and reinforcement was delivered on $50 \%$ of the trials, independently of the subjects' choices. In all other respects test trials were similar to training trials. The pigeons were tested for five consecutive test sessions. In each session, the pigeons were tested four times with white and blue samples at each of the seven durations, for a total of 56 trials per session. Training and test trials were presented in random order.

\section{RESULTS}

\section{Acquisition}

Acquisition to criterion (one session at $90 \%$ correct or better) data were analyzed with a two-way repeated measures ANOVA with sample duration $(2$ or $10 \mathrm{sec})$ and pecking requirement (pecking or not pecking) as factors. The pigeons learned the 2-sec sample trials (mean $=7.6$ sessions) significantly faster than they learned the 10-sec sample trials (mean $=14.9$ sessions) $[F(1,10)=7.24$, $p=.02]$, but neither the difference in acquisition on pecking (mean $=3.3$ sessions) and DRO trials (mean $=19.2$ sessions) $[F(1,10)=4.15, p=.07]$ nor the interaction of sample duration $\times$ pecking requirement $[F(1,10)=2.46$, $p=.15]$ was significant.

By the end of training, overall matching accuracy was high (94.7\%). A two-way ANOVA performed on the data from the last training session indicated that accuracy on pecking trials $(97.4 \%)$ was significantly higher than on DRO trials $(92.0 \%)[F(1,10)=9.99, p=.01]$. However, neither the effect of sample duration $[F(1,10)<1]$ nor the pecking $\times$ sample duration interaction $[F(1,10)<1]$ was significant.

Although by the end of training the pigeons were quite successful in meeting the sample-response requirement, on the last training session they still aborted an average of 9.2 pecking trials and $4.5 \mathrm{DRO}$ trials. Thus, in spite of the

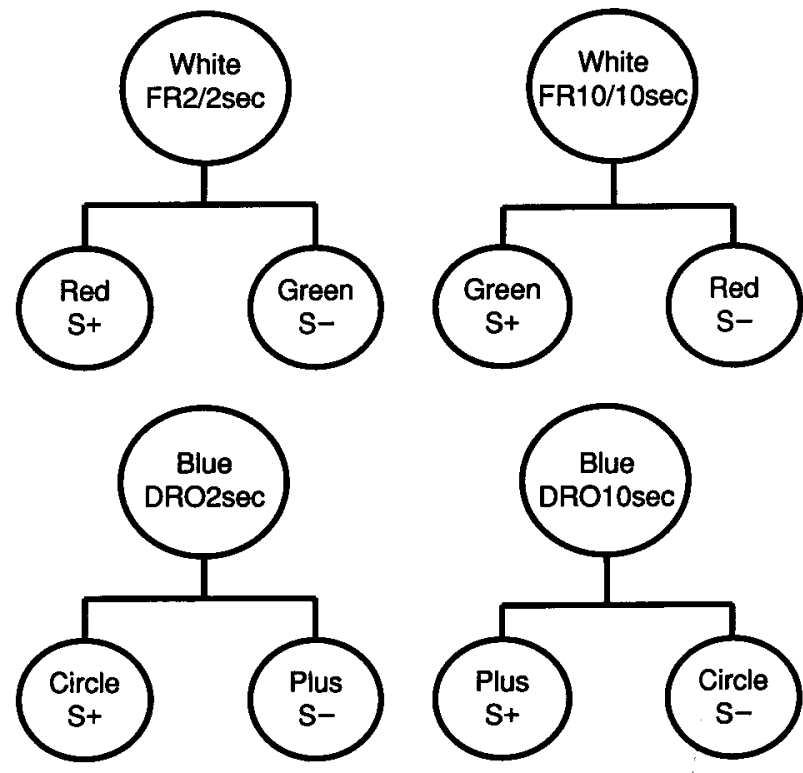

Figure 1. Schematic of the four training trial types for the pigeons trained to peck at white samples and to refrain from pecking at blue samples. White sample trials appeared for 2 or $10 \mathrm{sec}$, and the pigeons were required to make a minimum of 1 peck/sec. Pigeons received reinforcement when, following a 2-sec sample, they chose the red comparison stimulus and, following a 10-sec sample, they chose the green comparison stimulus. Blue sample trials also appeared for 2 or $10 \mathrm{sec}$, but the pigeons were required to refrain from responding for their entire duration (known as differential reinforcement for other behavior, or DRO). For the remaining pigeons, the response requirements associated with white and blue samples were reversed (schematic not shown). Red and green comparison stimuli still followed samples that required pecking; following short sample stimuli, red was correct $(S+)$, and green was correct following long sample stimuli $(\mathrm{S}+)$. Circle and plus comparison stimuli followed samples that required the pigeons to refrain from pecking; following short sample stimuli, vertical was correct $(\mathrm{S}+)$, and horizontal was correct following long sample stimuli $(\mathrm{S}+)$.

fact that matching accuracy on DRO trials was worse than on pecking trials, the DRO requirement was easier to meet than the pecking contingency. A two-way ANOVA was performed on the data from the aborted trials, with pecking (pecking vs. DRO trials) and duration (2- vs. 10-sec samples) as the two variables. Due to considerable variability among the pigeons, the effect of pecking was not statistically reliable $[F(1,10)=1.67, p=.22]$, nor was the effect of duration significant $[F(1,10)=1.74, p=$ .22]. In addition, the pecking $X$ duration interaction was not statistically significant $[F(1,10)<1]$.

\section{Testing}

The point of subjective equality was estimated for each pigeon in each condition by drawing a straight line between the longest sample duration for which choice of the "long" comparison was below $50 \%$ and the shortest sample duration for which choice of the "long" comparison was above $50 \%$ of the long responses, and then calculating the point at which that line crossed the $50 \%$ long responses line. The point of subjective equality on refrain-from-pecking 
trials was $4.20 \mathrm{sec}$, whereas the point of subjective equality on required-pecking trials was $5.18 \mathrm{sec}$ (see Figure 2). Thus, pecking resulted in a shift of almost $1 \mathrm{sec}$ in the point of subjective equality. A correlated $t$ test performed on the point of subjective equality for each pigeon for trials on which pecking was required versus trials on which pecking was prohibited indicated that the difference was statistically significant $[t(10)=2.86, p=.02]$.

We also calculated the difference limen (DL), the difference in sample duration between $25 \%$ and $75 \%$ choices of the long comparison, for samples that required pecking and those that prohibited it for each pigeon. For samples that required pecking, the mean DL was $3.1 \mathrm{sec}$. For samples that prohibited pecking, the mean DL was 3.9 sec. This difference was not statistically reliable $[t(10)=1.42$, $p=.19$ ].

During testing, matching accuracy on training trials for which pecking was required $(94.9 \%$ correct) and on trials for which pecking was prohibited $(85.8 \%$ correct) were both quite high, as was matching accuracy on 2-sec sample trials $(92.2 \%$ correct) and 10 -sec sample trials $(88.5 \%$ correct). An ANOVA performed on the training durations during testing indicated that the effect of pecking was statistically significant $[F(1,10)=20.37, p=.001]$; however, neither the effect of sample duration (training values) $[F(1,10)=4.65, p=.06]$ nor the interaction $[F(1,10)<1]$ was significant.

\section{DISCUSSION}

Pigeons were trained to discriminate between sample durations of 2 and $10 \mathrm{sec}$. On some trials they had to peck at the sample stimulus at a specified rate, whereas on other trials they had to refrain from pecking at it. On test trials,

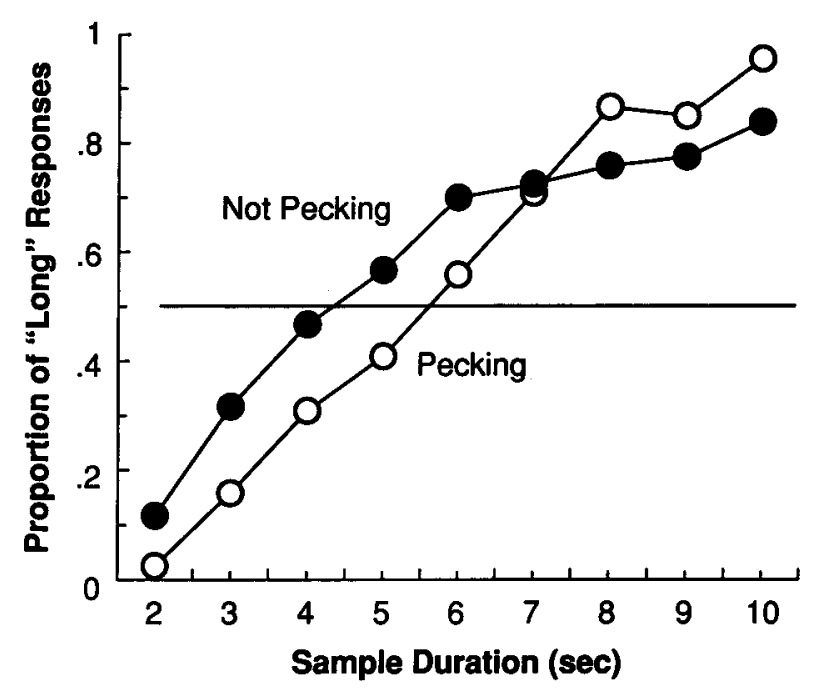

Figure 2. Proportion of instances in which pigeons chose the response button associated in training with the long (10-sec) sample as various sample durations between 2 and 10 sec were presented. The horizontal line represents equal choice of the two alternatives (the point of subjective equality). samples of intermediate duration were presented to determine the point of subjective equality on trials when the pigeons were pecking and when they were not pecking. When the pigeons were pecking at the samples, their point of subjective equality was almost $1 \mathrm{sec}$ longer than when they were not pecking.

A longer point of subjective equality suggests two possible mechanisms. One is that actual time was passing faster than subjective time. These results are similar to results from experiments in which human participants made timing judgments while under a cognitive load (Hoekstra, 2005). Enforced pecking may be analogous to cognitive load because the pigeons must maintain a relatively high rate of pecking to avoid having the trial abort.

The idea that cognitive load may affect the psychophysical test functions has also been examined by Sutton and Roberts (2002). Although they found some evidence that the point of subjective equality occurred later when the cognitive load was greater, there was also a general flattening of the psychometric function (i.e., generally poorer control by sample duration) when the cognitive load was greater. However, Sutton and Roberts required that their pigeons be able to choose comparisons, sometimes on the basis of the duration of the sample and at other times on the basis of the line orientation that was presented. It may be that if the cognitive load is too great, the generally poorer performance on the timing task can mask changes in the rate at which subjective time passes.

An alternative account of the shift in the point of subjective equality is that the start or stop times that the pigeons used to estimate the intervals differed depending on the type of trial. If on trials that required pecking the pigeons started timing later, this would also result in a shift in the point of subjective equality to longer sample durations. It may be that on trials that did not permit pecking, the pigeons began timing immediately; however, on trials on which the pigeons had to peck, they started timing only once they had begun pecking. In fact, consistent with the hypothesis that the start of timing was delayed on trials that required pecking, the slopes of the two psychophysical functions as measured by the DL were quite similar. Inconsistent with this hypothesis, however, is the crossover in the two functions at samples of $7 \mathrm{sec}$ (see Figure 2). In any case, if the pigeons did start timing later, this would be consistent with the hypothesis that requiring pecking adds to the cognitive load of the timing task.

Alternatively, it may be that the pigeons were simply less accurate on trials that did not require pecking because they were less attentive to the sample duration. Of course, being less attentive on those trials could also account for their poorer performance on short sample trials. But this would not account for the difference in the point of subjective equality, which should not be influenced by the level of matching accuracy on short and long sample trials.

Positing that pecking involves a greater cognitive load may seem strange, since matching accuracy was better when the pigeons were required to peck than when they were required to refrain from pecking. This paradox can be resolved by positing the presence of two different mech- 
anisms. First, matching accuracy may have been worse when the pigeons were required to refrain from pecking because in avoiding pecking the sample response button, they were also less accurate in detecting when the sample terminated. Such variability in sample offset would likely lead to a general decrease in accuracy. Second, the cognitive load produced by the pecking requirement either altered the subjective passage of time or delayed the start of timing, which in either case would lead to a systematic shift in the psychophysical function.

Although cognitive load has been posited to be the mechanism responsible for the shift in the point of subjective equality, shared attention may also be involved. When attention is directed away from duration judgments, either slower duration judgments or a delay in the start of such judgments may result.

Recently, Miki and Santi (2005) have reported that when pigeons judge the duration of dark versus light intervals, the dark intervals yield an earlier point of subjective equality. As pigeons typically peck at lit samples even when not required to and refrain from pecking at dark samples, these results are consistent with those of the present experiment. On the other hand, it is not clear how either cognitive load or shared attention can account for the effects of other manipulations. For example, Maricq, Roberts, and Church (1981) found that for rats, amphetamine consumption caused the point of subjective equality to shift to shorter sample durations, which suggests that the effect of amphetamine is to increase the subjective passage of time (but see Stubbs \& Thomas, 1974). Also, there is evidence that a decrease in stimulus intensity can result in a later point of subjective equality (Kraemer, Brown, \& Randall, 1995; Wilkie, 1987). Thus, decreasing the intensity of a timed stimulus appears to make time pass more slowly.

In the present experiment, we assumed that it was the cognitive load or shared attention associated with the required (relatively high) response rate that was responsible for the shift in the point of subjective equality, rather than the act of responding per se. One way to test this hypothesis would be to require that pigeons time intervals while performing a relatively difficult schedule, such as the differential reinforcement of low rates of responding (DRL), which requires little responding. With this schedule, a pigeon would have to space its responses to the short and long samples with a minimum interresponse time of, say, $1 \mathrm{sec}$ before the comparison stimuli would appear. Such a schedule should add to the cognitive load or shared attention without requiring many responses. If a DRL requirement also shifts the point of subjective equality to longer sample durations, it would offer support for the cognitive load hypothesis.
Whether shifts in the psychophysical function found in the present experiment result from a change in the production (or accumulation) of pulses or from differential latency of the start of duration judgments, the phenomenology associated with the subjective passage of time in animals is worthy of further research.

\section{REFERENCES}

Block, R. A., \& ZaKaY, D. (1997). Prospective and retrospective duration judgments: A meta-analytic review. Psychonomic Bulletin \& Review, 4, 184-197.

Chastain, G., \& Ferraro, F. R. (1997). Duration ratings as an index of processing resources required for cognitive tasks. Journal of General Psychology, 124, 49-76.

Chaston, A., \& Kingstone, A. (2004). Time estimation: The effect of cortically mediated attention. Brain \& Cognition, 55, 286-289.

Church, R. M. (1978). The internal clock. In S. H. Hulse, H. Fowler, \& W. K. Honig (Eds.), Cognitive processes in animal behavior (pp. 277 310). Hillsdale, NJ: Erlbaum.

GibBon, J. (1986). The structure of subjective time: How it flies. In G. H. Bower (Ed.), The psychology of learning and motivation (Vol. 20, pp. 105-135). San Diego: Academic Press.

GIBBON, J., \& CHURCH, R. M. (1984). Sources of variance in an information processing theory of timing. In H. L. Roitblat, T. G. Bever, \& H. S. Terrace (Eds.), Animal cognition (pp. 465-488). Hillsdale, NJ: Erlbaum.

HoEkstra, S. J. (2005, May). "Time flies when you're having fun": Cognitive load and perceptions of time. Paper presented at the meeting of the Midwestern Psychological Association, Chicago.

Kraemer, P. J., Brown, R. W., \& Randall, C. K. (1995). Signal intensity and duration estimation in rats. Behavioural Processes, 34, 265-268.

MaricQ, A. V., Roberts, S., \& ChURCh, R. M. (1981). Methamphetamine and time estimation. Journal of Experimental Psychology: Animal Behavior Processes, 7, 18-30.

MrKI, A., \& SANTI, A. (2005). The perception of empty and filled time intervals by pigeons. Quarterly Joumal of Experimental Psychology, 58B, 31-45.

PlatT, J. R., \& DAVIS, E. R. (1983). Bisection of temporal intervals by pigeons. Journal of Experimental Psychology: Animal Behavior Processes, 9, 160-170.

Roberts, W. A., Cheng, K., \& Cohen, J. S. (1989). Timing light and tone signals in pigeons. Journal of Experimental Psychology: Animal Behavior Processes, 15, 23-35.

STUBBS, D. A. (1976). Scaling of stimulus duration by pigeons. Journal of the Experimental Analysis of Behavior, 26, 15-25.

StuBbs, D. A., \& ThомAS, J. R. (1974). Discrimination of stimulus duration and $d$-amphetamine in pigeons: A psychophysical analysis. Psychopharmacologia, 36, 313-322.

SutTon, J. E., \& RoBerTs, W. A. (2002). The effect of nontemporal information processing on time estimation in pigeons. Learning \& Motivation, 33, 124-140.

WILKIE, D. M. (1987). Stimulus intensity affects pigeons' timing behavior: Implications for an internal clock model. Animal Learning \& Behavior, 15, 35-39.

ZAKAY, D. (1993). Time estimation methods: Do they influence prospective duration estimates? Perception, 22, 91-101.

(Manuscript received November 21, 2005; revision accepted for publication March 7, 2006.) 